\title{
Chapter 10 \\ Hidden Connections and Double \\ Meanings: A Mathematical Viewpoint of Affective and Cognitive Interactions in Learning
}

\author{
Inés M. Gómez-Chacón
}

\begin{abstract}
This paper poses methodological questions concerning the evaluation of emotion in the process of mathematical learning where the interaction between emotion and cognition occurs. These methodological aspects are considered not only from the perspective of educational psychology but from that of mathematics education. Some epistemological and ontological aspects, which are considered central to the cognition-affect interplay, are noted. Special attention is given to the notion of cognitive-affective structure as a dynamic system. The interplay between cognition and affect in mathematics is viewed through the concepts of local and global affect and using a mathematical working space model. A model of this interplay is illustrated with research examples, enabling us to move from descriptions of cognition-affect at an individual level to the explanation of the tendency of a group. The non-linear modelling of emotion is reflected in the affect-cognition local structure.
\end{abstract}

Keywords Structures of affect - Affective and cognitive interactions Epistemology and emotions - Mathematical working space $\cdot$ Learning

\subsection{Introduction}

Current advances in philosophy of science (Brun et al. 2008), social neuroscience for education (Immordino-Yang and Damasio 2007), and cultural approaches in social psychology (Harré 2009) have been highlighting interconnections between cognition and emotion, which frequently allow for emotions to contribute to the growth of knowledge. The demand for research that identifies these interconnections derives from the benefit that knowledge about them can impart to the design of learning environments.

I. M. Gómez-Chacón ( $\square$ )

Universidad Complutense de Madrid, Madrid, Spain

e-mail: igomezchacon@mat.ucm.es

(C) The Author(s) 2018

G. Kaiser et al. (eds.), Invited Lectures from the 13th International Congress

on Mathematical Education, ICME-13 Monographs,

https://doi.org/10.1007/978-3-319-72170-5_10 
Responding to this need to characterize these interconnections, we focus on specific mathematical knowledge. This chapter highlights some precise aspects of the epistemological and ontological dimension that this analysis of the cognition-affect interplay entails. We consider that the epistemic meaning of the emotions must be studied according to the specific characteristics of the epistemology of mathematical knowledge and under a dynamic interrelation approach (a dynamic system of affect). Recent reviews of affect (e.g., Pepin and Rösken-Winter 2015) have highlighted the need to make explicit the combined nature of cognition, motivation, and emotion on mathematical work in dynamic affect systems. Here, we propose viewing these systems in a holistic manner. This means an understanding of a system by examining the linkages, interactions, and relationships between the elements that compose the entirety of the system. Typologies of cognition and affect structures are identified. In describing these structures in our empirical studies, we found characterizations of affect that depend on the nature of the context (local-global) and on the kinematics of the mathematical processes involved (static-dynamic) as well as on the dynamic movement of the nature of the phenomenon at an individual or group level. Some dynamic affect systems may have properties that can only be studied at the higher emergent level in a group, so patterns of interaction that characterize the affective systems of individuals and collectives need be observed.

Specifically, we are looking to make progress in the understanding of the following aspects of the interaction between cognition-affect in mathematics:

(a) The conceptualization of structures of this interaction;

(b) The affective-cognitive reference system given by specific mathematical knowledge, which includes the distinction between mathematical knowledge and appraisal processes; and

(c) The complexity of explicitness of dynamic systems involved in this interaction, from the uniqueness of the individual patterns of reasoning to the characterization of the tendency of a group.

Section 10.2, Theoretical Fundamentals, deals with some aspects of what epistemologically characterizes the affective-cognitive system in mathematical reasoning and considers a model of analysis with its own categories. Sections 10.3 and 10.4 present the study by which the local affect-cognitive structure has been determined, using this model of analysis. A final section addresses the conclusions of the study and makes suggestions for developmental aspects in future research.

\subsection{Theoretical Fundamentals}

In order to tackle the intricacy of the subject under investigation, a number of theoretical considerations are employed to establish a consistent interpretative framework: epistemological dimension and the affective-cognitive reference system model. 


\subsubsection{Affective-Cognitive Reference System: The Zig-Zag Path in Mathematical Reasoning}

We begin with the assumption that no affective behavior is devoid of cognition. Some authors use terms such as affective schemes or cognitive-affective schemes (Schlöglmann 2005) in an attempt to study this interaction in greater depth. Far from contradicting the aforementioned basic assumption, the acknowledgement of "affective structures" confirms that they are isomorphs of cognitive structures and the result of intellectualization (Piaget 1981), which exists whenever feelings are structured. In fact, structure and the workings of cognition and affect are indivisible in all behavior. Nevertheless, I note that there are authors such as Goldin (2000) who see this interaction as a representational system; in his works he leans more towards a separation of structures of affect and cognition, following the work of Zajonc (1980).

Maintaining a dialogue concerning cognition-affect interaction entails bearing in mind matters relating to the singularity of individual reasoning patterns as well as social interaction. An initial insight is that mathematical reasoning does not follow a straight line but, as Lakatos (1976) contends, a zig-zag course.

Discovery does not go up or down, but follows a zig-zag path: prodded by counterexamples, it moves from the naïve conjecture to the premises and then turns back again to delete the naïve conjecture and replace it with the theorem. Naïve conjecture and counterexamples do not appear in the fully fledged deductive structure: the zig-zag of discovery cannot be discerned in the end-product. (Lakatos 1976, p. 42)

Affect is essential to the self-regulation and self-reflection that takes place in the course of reasoning. Self-assessment of personal competence, affective response, and self-regulation are keys to problem solving. In this regard, Lakatos notes that perseverance is needed to surmount the cognitive and affective difficulties arising in "conscious guessing, because it comes from the best human qualities: courage and modesty" (Lakatos 1976, p. 30).

\section{Motion and e(motion)}

The epistemological view of Lakatos challenges a simplistic view of objectivity in mathematical knowledge. In each theory (scientific or mathematical), the subjective dimension, either as a psychological process or a sociological process, is inexorably involved. For Lakatos, mathematical thinking does not develop monotonically: "Informal, quasi-empirical, mathematics does not grow through a monotonous increase of the number of indubitably established theorems but through the incessant improvement of guesses by speculation and criticism, by the logic of proofs and refutations" (1976, p. 5) in a dynamic zig-zag motion (trajectories).

In this characterization of mathematical knowledge, motion (hence, e(motion)) is essential. The word (e)motions or simply motions are used to denote mental states not fully described by a formalized language: They are individual instances of subjective, conscious experience. The "motions of the cognitive-affective interplay" are inner motions, interior movements consisting of thoughts, imaginings, emotions, inclinations, desires, feelings, repulsions, and attractions. To identify this 
interaction involves becoming sensitive to these movements, reflecting on them, and understanding where they come from and where they lead us. The word motion conveys the movement and purpose inherent in the meaning of the word emotion; in contrast, the word emotion conveys only a sense of spontaneous change and movement without a definite purpose.

In this light it may be said that the continuous process underlying motion holds and connects the stages that occur in the movement of problem solving. We invite the reader to consider the phenomenon of interaction as a key element of this movement. Moreover, affect is considered not only as energy (in the sense in which Piaget distinguished between affectivity as energy and cognition as structure) but as a structure, although a structure that is not static but dynamic (see Sect. 10.2.2).

\subsubsection{Affective-Cognitive Reference System Model}

Since our first study in 1997 on affect and cognition (Gómez-Chacón 2000a, b; Gómez-Chacón and Figueral 2007), we have been interested in the methodological aspects that would allow us to establish an analytical model. In this respect our major claim is that in order to understand cognitive-affect interplay in the acting individual at a particular moment, it is necessary to attain knowledge of that individual at different levels: individual, group, society, and integrally as holistic. This knowledge would capture aspects that model the dynamics of cognitive-affect using some constructs: structures of (local and global) affect, cognitive dimension (valuation processes and mathematical processes), and meta-affect.

\section{Conceptualization of affect and structure}

Prior to describing these constructs (categories) we need to clarify the meaning of the terms affect and structure.

We wish to note that the term affect has a different meaning if it is used by educational psychology or by mathematics education. Pekrun and Linnenbrink-Garcia (2012) note that the term affect in psychological emotion research refers more specifically to emotions and moods; here, we use the term in the broad sense prevalent in mathematics education (e.g., Goldin 2014; Pepin and Rösken-Winter 2015). We share the view of those who within mathematics education regard the importance of emotions as being partly or mainly through their connection with attitudes, beliefs, and values.

Affect is understood here as a notion of a higher order that includes all of the above as a phenomenon. Affect is defined as a "quality power status variable", with duration and intensity at the level of consciousness.

Regarding the meaning of structure, in every structure definition, the concept structure refers to following elements: (a) a whole, (b) the parts of this whole, and (c) the relationship between these parts. In the 50s, mathematical structure was defined as "a specific set of relations or laws describing the functions of a phenomenon that can be represented by a model" (Bastide 1962, p. 14). In this light, 
we understand structure as a system of interconnected elements in which a change in one element necessarily causes changes in other elements. This type of approach has been expressed in positions such as that of Piaget in the analysis of mathematical structures, defining structure as a system of transformations, which cites fullness (a whole), transformation, and self-regulation as important characteristics. The phenomenon of invariance is associated to the transformation. For many structuralist thinkers, this principle of invariance appears as a key element.

In our case we seek to confirm specific patterns, specific cases, and simple rules that give a typical structure or rhythmic sequences in this interaction between cognition and affect in the individual and explore whether these same patterns are extended to a group of individuals. The structure definition that we use here is dynamic (a dynamic system), and it corresponds to the predominant use of the term today.

We consider that the importance of capturing structure models, as already mentioned, or what other colleagues have expressed as "cognitive-affective schemes," resides not only in identifying schemes but also in the potential created for the recursive construction for the understanding of that scheme. Many of the individual actions are performed unconsciously. However, many of the operations through which we assemble our experiential world can be explored and the knowledge attained can help make learning different, and perhaps better.

Structures of affect: local and global

Local affect-cognition structure occurs at the micro and individual level. It is defined as the understanding of the affective reactions of students towards mathematics by observing and knowing the stages in the process of change of emotional reactions during problem solving and detecting cognitive processes associated with positive or negative emotion. It consists in representing the information on emotional reactions that have an impact on conscious processing. This allows us to establish productive affective pathways. Affective pathways are sequences of (local) emotional reactions that interact with cognitive configurations in problem solving.

Global affect-cognition structure occurs at the medium and macro (individual, group, and society) level and is understood as a result of these factors:

1. The summary of the pathways followed by the individual in the local affective dimension. These pathways are established with the cognitive system and they contribute to the construction of the general structures of one's self-concept as well as the beliefs about mathematics and the learning of mathematics.

2. The interactions and social-cultural influences on individuals and how that information is internalized and shapes their belief systems. Two aspects to take into account: the social representations of mathematical knowledge and the socio-cultural identity of subjects. The features that the students' identities have in their context are equivalent to a network of meanings that will be manifested in the learning of mathematics. These meanings will throw light on our search for a greater understanding of the global configuration of the affective aspect, their way of knowing and reacting affectively to the learning of mathematics, and their way of constructing belief systems and the knowledge of these. 
To sum up, the term local affect includes emotional states and mood states but also their moment-by-moment interactions with cognition, the social environment, the emotions of others, and the individual's traits. Global affect includes trait emotions as well as stable structures that incorporate emotions - not only attitudes, beliefs, and values but constructs such as mathematical self-identity (Gómez-Chacón 2000a, b; Gómez-Chacón and Figueral 2007).

\section{Cognitive dimension}

In this study we use the term cognitive broadly. On the one hand, this refers to the extensive use of processes of evaluation (cognitive appraisal; Lazarus 1991) and, on the other hand, to the characterization of the subjects' personal meanings of the cognitive dimension of the heuristic that acts in the solution of problems (mathematical cognitive processes) (Goldin 2004; Gómez-Chacón 2000a, b, 2015; Schoenfeld 1994).

Certain distinctions between "knowledge" and "evaluation/appraisal" are essential in our study. The need for this differentiation arises not only from our studies but from studies that have expressed the need to make a distinction between knowledge and appraisal (Lazarus 1991). We consider that there are certain features with emotional implications that may be particular to mathematics as compared with other school subjects, and they are related to the cognitive dimension. For instance, the circulation between different types of mathematical reasoning (discursive, instrumental, and visual) and the difficulty of transitions between them or the necessity of formal language, which involves specific semiotic systems and representation. As we will present in Sect. 10.3, the analysis of the cognitive dimension of mathematical work requires a specific model. In this section we present the mathematical working space framework (MWS) model in order to go deeper into the analysis of local affect and cognition interplay.

We can also speak to the differentiation of cognitive demands according to concepts of the difference between mathematical knowledge in general and contextual mathematical knowledge. The former includes establishing attitudes, beliefs and intuitions about oneself, while the latter is active in a particular situation or with a specific content whose impact on emotion can be very different. Regarding cognitive appraisal, this will be referred to as the generation of personal meanings and how the valuation of that knowledge makes it potentially emotional, i.e., how the situation globally affects relevance for the person, in relation to the goals, and to resource management.

\section{Meta-affect or meta-emotion}

Notions such as 'meta-affect' or 'meta-emotion' are required to refer to affect about affect or to affect in cognition that is about affect. In this way they serve to monitor affect both through cognition and affect. It's referred to meta-emotional understanding and meta-emotional skills. It shows how meta-affect arises in the formation of an individual's cognitive and affective schemes (Gómez-Chacón 2000a, b, 2015; DeBellis and Goldin 2006; Malmivuori 2006; Schlöglmann 2005).

Cognitive understanding of affect enables individuals to control their actions in affective situations. Successful handling of affective situations stabilizes affect schemata and consequently beliefs through simulation as a cognitive window to 
emotions. Prior research has shown that the stability of an individual's beliefs is closely related to the interaction among belief structures. These include not only affect (feelings and emotions) but also and especially meta-affect (emotions about emotional states, emotions about cognitive states, thinking about emotions and cognitions, and regulation of emotions; Gómez-Chacón 2000b). These findings reveal the personal and social dimensions of the affective constructs and self-control of emotions.

The section that follows deals with determining the local affect-cognition structure. The main reasons for doing so are that the local affect-cognition structures offer a profile of global affect structure of the subjects and also because, as we indicated at the beginning, the results of this basic research could be easily integrated in classroom practice.

\subsection{Determining the Local Affect-Cognitive Structure}

To empirically illustrate this section we will take a recently developed study about affective pathways and interactive visualization in the context of technological and professional mathematical knowledge (Gómez-Chacón 2012, 2015). For a period of three years, a study with university students with degrees in mathematics who were possible future secondary school teachers (98 students, 65 female and 33 male) was carried out. In this study, a teaching experiment was developed through problem solving. A questionnaire was composed of six non-routine problems about geometric locus to be solved using GeoGebra. The problems required the solver to use a proposed chain of various steps of visual processing (technical, deductive, and analytical) in order to find the solution. Each of the 98 students solved the six problems. In order to identify both types of cognitive processes and emotions, data were collected from the subjects' problem-solving protocols as well as with two questionnaires: one on beliefs and emotions about visual reasoning completed at the outset and another on the interaction between cognition and affect in a technological context filled in after each problem was solved.

As introduced in Sect. 10.2.2, when we focus on the local affective-cognitive interaction as a research goal, we are trying to "capture" (explain and model) the precise mathematical elements of both cognitive processes and appraisal processes; patterns, routines, and dynamic changes in the affective pathways of each individual; and the transition of local affective-cognitive local structure to global affective-cognitive structure in the individual. Explaining and modelling these specific aspects involves both methodological and theoretical options. To describe these aspects and illustrate them with the results of empirical research the following points were established:

(a) Considerations for the analysis of the cognitive mathematical dimension (Sect. 10.3.1)

(b) Modeling the local structure of affect in the individual: routines and bifurcations (Sect. 10.3.2)

(c) Modeling local affect structure in a group (Sect. 10.4). 


\subsubsection{Considerations for the Analysis of the Cognitive Mathematical Dimension}

Following the term cognitive described in Sect. 10.2, two aspects need to be characterized: the cognitive dimension of the mathematical visualization processes and the processes of cognitive appraisal.

Relative to the cognitive dimension, the mathematical working space (MWS) model (Gómez-Chacón and Kuzniak 2013; Gómez-Chacón et al. 2016), together with the instrumental dimension, are used to describe the complexity involved in applying technology to the mathematical and cognitive aspects of geometric tasks. Within the mathematical working space framework, cognitive and epistemological levels need to be articulated to ensure a coherent and complete geometric work.

Epistemological and cognitive levels are defined in terms of three genesessemiotic-figural, instrumental, and discursive (see Fig. 10.1) - to guarantee complete and consistent geometric work. The cognitive plane is introduced to describe the cognitive activity of a single user. In this model, the idea of three cognitive processes involved in geometrical activity is adapted from Duval (2005): (a) a visualization process connected to the representation of space and material support, (b) a construction process determined by instruments (ruler, compass, etc.) and geometrical configurations, and (c) a discursive process that conveys argumentation and proofs. Both planes, cognitive and epistemological, need to be articulated in order to ensure a coherent and complete geometric work. This articulation assumes the presence of some transformations that are possible to define through three fundamental geneses represented in the diagram below (Fig. 10.1).

In the analysis of the problem solution we examined some key aspects of how both figural and instrumental developments are involved in the learning process in a computer environment. An understanding of the visualization processes identified which ones are associated with patterns of use, with structuring information by sign operations, or with a heuristic function that allows the user to anticipate and plan actions and modes of validation.

The study of the local affect-cognitive structure addresses the so-called intra- and inter-plane zig-zag paths in mathematical reasoning. These epistemological aspects of this point were introduced in detail in Sect. 10.2. There the conception of interaction cognition and the complexity of treating such interaction were expressed. As additional information, the interested reader can consult Gómez-Chacón et al. (2016), where the nonlinearity or zig-zag motion was given in detail, with an example of the interaction between demonstrative reasoning and mathematical attitudes by two secondary school students.

Regarding zig-zag paths in mathematical reasoning, in the presented study we focus on the plane that is associated with figural and instrumental genesis. Movements comprising inter-genesis and intra plane transitions were analyzed at the local level (see Table 10.1). Image typology and the use of visualization were analyzed as well. They are categorized conceptually: the use of visualization as a 


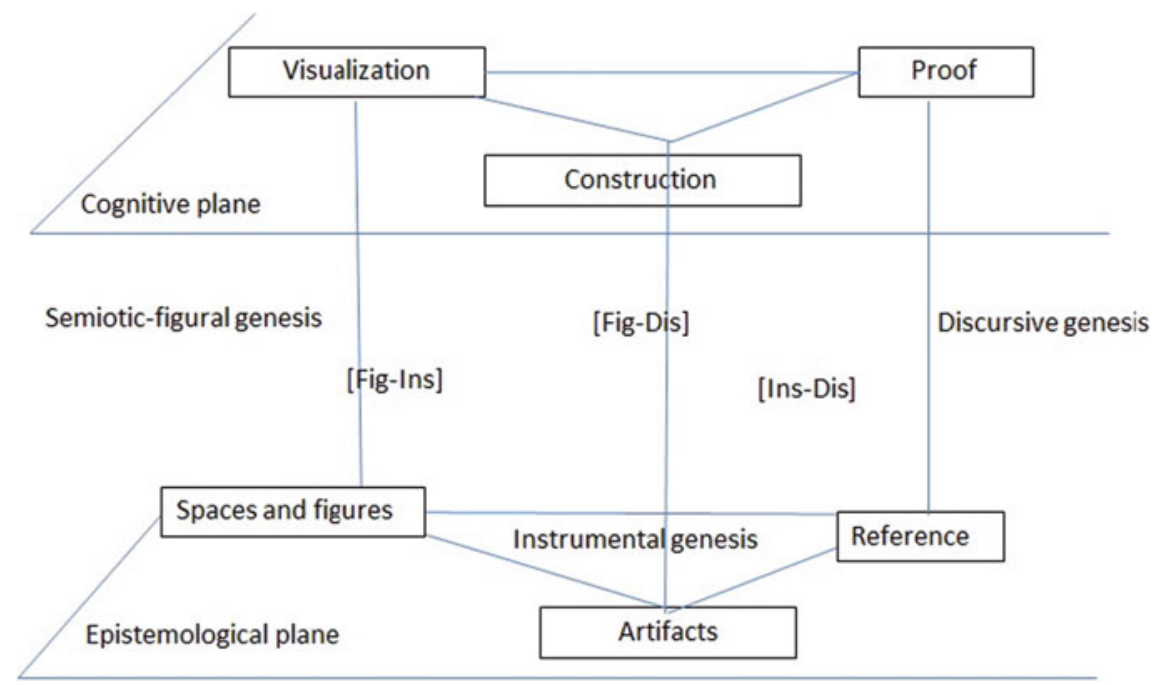

Fig. 10.1 Geometric working space, geneses, and vertical planes in an ideal MWS

reference and its role in mathematization and the heuristic function of images in problem solving.

Finally, referring to the processes of cognitive appraisal, different patterns of appraisal are analyzed (pleasantness and goal-path obstacle) and it is determined whether a given belief (beliefs about visualization and beliefs about technology) can elicit different emotions from different individuals.

\subsubsection{Modeling the Local Structure of Affect in the Individual: Routines and Bifurcations}

Establishing patterns of interaction of cognition and affect requires analysis at the microscopic level of individuals and all data sources. Below we present an analysis in the case study for the following problem:

The ladder: The top of a 5-m ladder rests against a vertical wall, and the bottom rests on the ground. Define the locus generated by midpoint $M$ of the ladder when it slips and falls to the ground. Define the locus for any other point on the ladder.

This analysis will endeavor to answer these research questions: What kind of cognitive affective pathways can be described? What are the influences that helped these students to stay on - or get back on - an enabling pathway of affect instead of sliding down to anxiety, fear, and despair? 


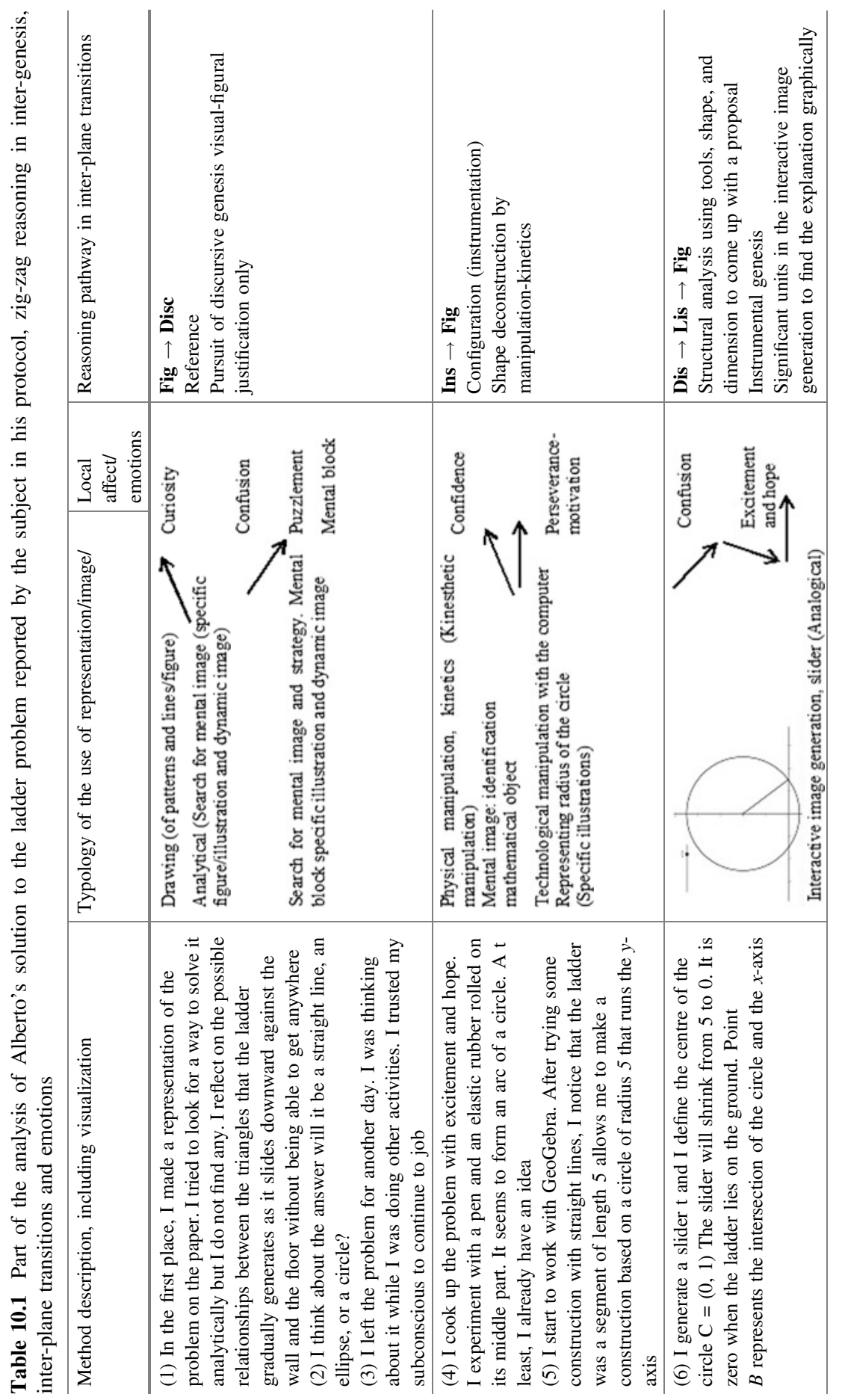


A possible establishment of patterns was analyzed for each subject:

1. Exposed beliefs and beliefs in action about visual thought and emotional reacting that can be generated.

2. Coincidences in the typologies of use of visualization and associated emotion.

3. Valuation made about the events that stimulate feelings: local affect. In this case, we have concentrated on processes related to visualization and technological use and the zig-zag reasoning in inter-genesis and inter-plane transitions: Figurative $\rightarrow$ Instrumental $\rightarrow$ Discursive (Fig $\rightarrow$ Ins $\rightarrow$ Disc).

We will use Alberto's Case as an example. Alberto is university student with a mathematics degree and is a visualizing individual. His enjoyment of mathematical visualization is closely intertwined with the evolutionary conception of mathematics. He considers visual reasoning to be essential in problem solving. He defines his own pathway of affect-cognition for the ladder problem (Fig. 10.2).

In Table 10.1, we have an extract of the cognition-affect relations in the problem according with the theoretical frame, where relationships between affect, cognition, and epistemological consideration are evidenced. An in-depth analysis of the problem-solving protocol for this exercise and the affective-cognitive pathway reported showed that this subject was able to describe and control emotions and identify causes. Three types of affective perspective were identified. First, Alberto always tried to find an answer even when in doubt or blocked. Alberto was continuously active, which is one way that many students cope with stress. Second, he was able to walk away from the problem, aware of the role of the subconscious in mathematics (See number (3) in first column of Table 10.1). Third, he struck a balance between the combination of graphic geometric thought and analytical task solving (transitions between Dis-Ins planes). These three behaviors were indicative of interaction between the cognitive-affective system and self-control. The description of emotions revealed that, from both a mathematical and a technical-instrumental perspective, self-confidence, stimulus, and joy were associated with the reproduction of physical forms and the visual/perceptive control implicit in a command of ancillary mathematical objects.

This type of microscopic analysis was performed for the whole teaching experiment (six problems). This allows each student to describe pathways in different problems. Comparison of the pathways of the six problems (Table 10.2) in the teaching experiment allows us to model the local structure of affect in the individual that shapes a more stable structure we call the global structure.

$$
\begin{gathered}
\text { Curiosity } \rightarrow \text { Confusion } \rightarrow \text { Puzzlement /Mental block } \rightarrow \text { Confidence } \rightarrow \text { Perseverance- } \\
\text { motivation } \rightarrow \text { Confusion/Exhilaration } \rightarrow \text { Confidence } \rightarrow \text { Confidence -Joy } \rightarrow \\
\text { Joy } \rightarrow \text { Perceived beauty } \rightarrow \text { Satisfaction } \rightarrow \text { GLOBAL AFFECT }
\end{gathered}
$$

Fig. 10.2 Affective pathways reported for the problem by Alberto 


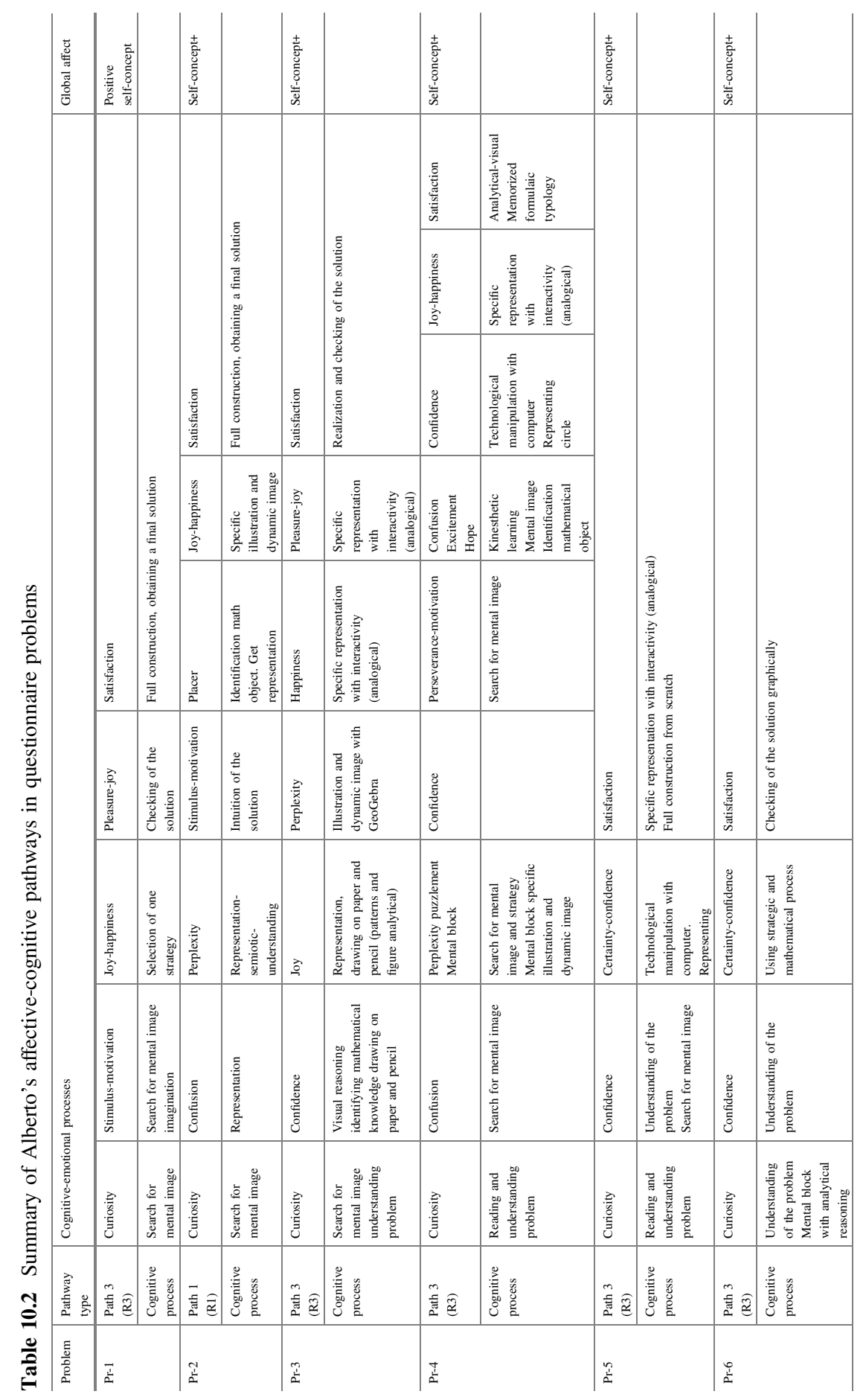


In Alberto's case, it can be seen that the cognitive processes of visual reasoning and negative emotion interaction occur in identifying strategies of interactive representation and processing of certain representations, where the student has to put into play the identification of parametric variations. He is a student who has a fluid use of images: concrete, kinesthetic, and analogic. This student recognizes an overall positive self-concept structure when working with computer mathematics.

In short, this kind of analysis allows us to identify patterns in the individual and between individuals in relation to their local cognitive affective structure:

1. In the individual, the summary of the pathways followed by the individual in the local affective dimension in different problems allow us to identify invariance and variances that occur in their local structure and that shape a more stable structure we call global structure.

2. Among individuals, variations in local structure according to individuals. This type of data analysis allows us to identify in-depth profiles of students with varied characteristics: gender, achievement in mathematics, beliefs, display style, and emotions. The affective pathways they reported were compared in order to glean information on meta-emotion and visualization. The comparison revealed: (a) the use of visualization and associated emotion and (b) the dependence of their emotional self-control on their individual perception, which was influenced by style, disposition, type of activity or skill, instrumental command, and belief system around technology-aided mathematical learning. For more detailed information see Gómez-Chacón (2012, 2015).

\subsection{Modeling Local Affect Structure in a Group}

In Sect. 10.3 we have focused on the understanding of the cognition-affect interaction in the individual. In this section we are going to take a step further: we will try to see how to make the leap from the characterization of individuals to the characterization of the group. To carry out this characterization, we ask the following questions: What are the differences in a subject's choice of pathway? What information on meta-emotion and visualization can be gleaned from the productive affective pathways reported by students in locus problems? Of these, which allow characterization of the tendency of a group?

Methodologically, in the affect dimension the characterization of a group has been solved by quantitative studies, mainly based on surveys. Here, we would like to raise other methodological forms that are based on qualitative measures and on quantitative behavior modeling collected in a qualitative way. In our most recent work we have worked with implicative statistical analysis models or models based on fuzzy logic (Gómez-Chacón 2015, 2017). In this paper I describe the first models. 


\subsubsection{Implicative Data Analysis}

In this research, together with the qualitative analysis (Sect. 10.3.2), an implicative analysis (Gras et al. 1997) was performed in order to explore the structure in cognition and affect interactions for the group. Gras's implicative statistical method has been conducted by using software called CHIC (Classification Hiérarchique Implicative et Cohésitive). At the descriptive level, it can be used to detect a certain degree of stability in the structuring, while for predictive purposes, it provides the grounds for assumptions. This statistical analysis was then used to establish rules of association for data series in which variables and individuals were matched in order to define trends in sets of properties on the grounds of inferential, non-linear measurement.

\section{Defining categories}

Two types of analyses were conducted in this study. The first was exploratory, descriptive, and interpretational, involving mainly inductive data analysis, with categories and interpretation building on the information collected (Sect. 10.3.2). This analysis used a qualitative approach based a cross-check of the solutions performed by three researchers. The following categories were defined:

1. Emotion associated with visual reasoning in the ladder exercise: P4EviP (like), P4EviN (dislike), P4EviM (mixed emotions), and P4viInd (indifferent).

2. Instrumental difficulties: The focus in this category was on two types of difficulties arising around the six problems (Fig. 10.3). Typology 1: Static constructions (discrete) (DT1P4). Typology 2: Incorrect definition of the construction (DT2P4).

3. Initial problem visualization: VisiP4.

4. Beliefs about visual reasoning: BeviP (positive), BeviN (negative).

5. Preferences and emotions around the use of visualization: EviP (like), EviN (dislike), EviInd (indifference).

6. Beliefs about computer-aided learning: BeGeoP (positive), BeGeoN (negative).

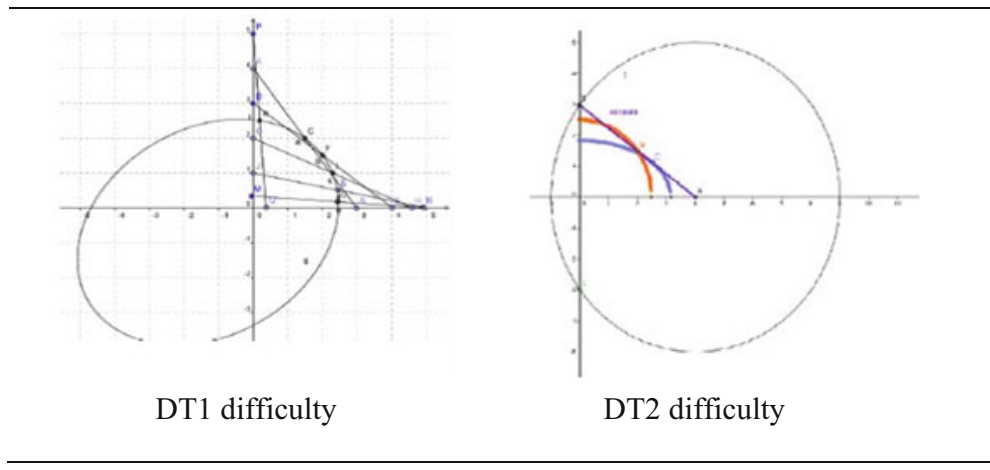

Fig. 10.3 Examples of subjects' difficulties with the ladder exercise 
7. Emotions concerning computer use: EGeoP (like), EGeoN (dislike), EGeInd (indifference).

8. Affective-cognitive pathways: R1 (mainly positive emotions), R2 (mainly negative emotions), R3 (subject-formulated, as described in Fig. 10.2 and Table 10.2).

All categories were compiled and coded in a matrix for implicative analysis performed using CHIC software. The identification of possible links among affective-cognitive pathways, emotions, and meta-emotion was the subject of the analysis.

\subsubsection{Results of the Modeling of Local Affect Structure in a Group}

In this study, a similar response was received when the beliefs explored related to the use of dynamic geometry software as an aid to understanding and visualizing the geometric locus idea. All the subjects claimed to find it useful, and $80 \%$ expressed positive emotions based on its reliability, speedy execution, and potential to develop their intuition and spatial vision. They added that the tool helped them surmount mental blocks and enhanced their confidence and motivation. As future teachers they stressed that GeoGebra could favor not only visual thinking, but help maintain a productive affective pathway. They indicated that working with the tool induced positive beliefs towards mathematics itself and their own capacity and willingness to engage in mathematics learning (self-concept as a mathematics learner).

Table 10.3 summarizes the frequencies of pathways and emotions associated with visualization in the ladder problem. Mixed affective pathways were identified, with alternating negative and positive emotions and optimized self-control of emotions.

The question that was posed to study the mix of emotions and meta-emotion in greater detail was "What are the differences in a subject's choice of these three pathways?" The preliminary analysis showed that pathway R3 was largely self-formulated and contained a much greater mix of emotions (Sect. 10.3.2). In most cases, moreover, the trend was not as explicit as in R1 (positive) or R2 (negative). Rather, negative feelings (which were controlled) were attributed to certain stages of the visualization process and positive feelings to success in representing the desired images. A hierarchy study of R3 yielded some significant affective-cognitive implications respecting visual processes: R3P4 $\rightarrow 0.99 \mathrm{VisiP} 4$ and R2P4 $\rightarrow 0.90 \mathrm{DT} 2 \mathrm{P} 4$.

Table 10.3 Percentage of affective-cognitive and emotional pathways associated with visualization in the ladder exercise

\begin{tabular}{l|l|l|l|l|l|l|l}
\hline & R1 & R2 & R3 & EviP & EviN & EviM & EviInd \\
\hline Problem (\%) & 46 & 12 & 40 & 18 & 25 & 53 & 3 \\
\hline
\end{tabular}


Fig. 10.4 Hierarchy tree

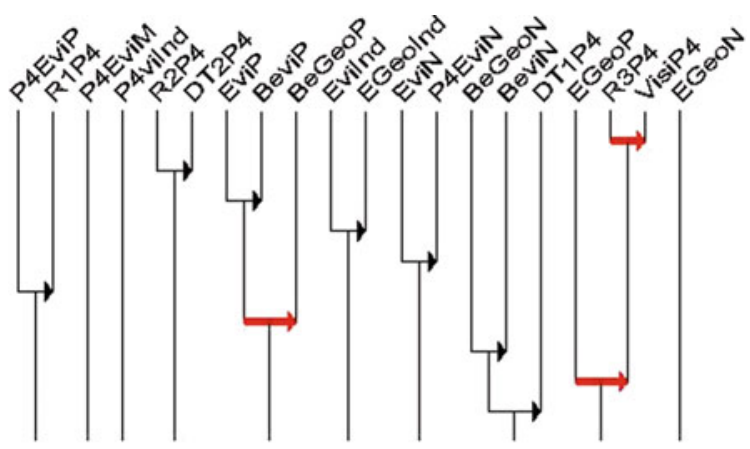

Three of the nine nodes obtained in the hierarchy tree were significant and identified the following groups (Fig. 10.4).

Group $1(\mathrm{~N}$ (level 1, cohesion: 0.998$)=(\mathrm{R} 3 \mathrm{P} 4 \mathrm{VisiP} 4))$, comprising over $40 \%$ of the initially visualizing subjects (in Problem 4) who indicated pathway R3 as the expression of their cognition-affect interaction. The most significant characteristic of these individuals was their positive feelings towards computers (use of GeoGebra (EGeoP) software).

Group 2 (N (level 7, cohesion: 0.276) $=(($ EviP BeviP) BeGeoP $))$, where the most prominent finding was that a belief in the use of GeoGebra was attendant upon a belief in and a preference for visual reasoning.

\subsection{Conclusion}

It has been argued, given the empirical evidence, that the link between cognition and affect is at the basis of the whole of mathematical activity. It has been noted, however, that similar investigations on the agenda of mathematics education are still very scarce. One of the main reasons for this shortfall is that it is difficult to carry out studies on affect: It is a question of the adequacy of theoretical and methodological frameworks.

We have tried to envisage both the epistemological and ontological keys inherent in the methodology designed to capture the interaction of cognition and affect in mathematics. Also, a study and research design has been shown that has resulted in an instrument that is significant and productive in addressing this goal.

The results of the research presented in Sects. 10.3.2 and 10.4 have shown the following findings: (1) Both cognitive mathematical processes and appraisal processes are key dimensions that explain the interplay between cognition and affect, and (2) the non-linear modeling of emotion is reflected in the affect-cognition local structure. It has been shown that the emotions in problem solving are not static. 
They are dynamic events that can be on-going throughout a problem-solving session. The inspiration of the nonlinear model of emotion described here is based not only on socio-constructivist approaches (such as Mandler's theory; Mandler 1984) but on other emotion theories with a more holistic view of the individual or a perspective systemic of affectivity that we presented in Sect. 10.2.

Regarding the connections between cognition and emotion, two categories seem to appear within the study: emotions that have causes known by the person who experiences them and words that are used to denote the causes of the emotions. The analysis of these connections sought not only to determine the relations between variables but also to develop rule models as well as use a description of qualitative data that can enrich this relationship. Notice that the analysis of the group (Sect. 10.4) as a multiple variable analysis with the capability of decision trees enables us to go beyond simple one-cause, one-effect relationships and to discover and describe things in the context of multiple influences.

The results of studies where non-linear modeling of emotion is reflected in the affect-cognition local structure drive us to formulate an open question about the type of generated structure. We might ask whether or not the aforementioned affective-cognitive interactions have a so-called fractal structure; that is, are they basically similar (self-similar) at the mental and subjective, micro-social (interpersonal or small-group), and macro-social (international or intercultural) levels? As is well known, fractality is a property of a great number of natural and cultural phenomena which are intensely studied by dynamic systems theory (formerly called chaos theory) and of many biological, demographic, and economic processes. Fractal structure is generated by basically similar dynamics (algorithms) on different levels of functioning. The result is a characteristic so-called self-similarity (or scale-independence) of structural patterns on different levels of complexity. The notion of fractality permits a methodologically correct transfer of small-scale (e.g., individual or interpersonal) observations to large-scale (e.g., inter-group or international) processes and vice versa.

Notice that studies on the exploration of fractal structures have been developed in the understanding of mathematical concepts, but we do not meet them in the field of interplay of cognition and affect (e.g., Singer and Voica 2010). So, although the present study casts some light on this issue of the fractal structure, it is still a question open to debate. The discussion on fractality can be established in the global-local cognitive-affective perspective taken in this paper and in the methodological transfer of small-scale observations to large-scale processes.

In the results of the described study, a type of fractal construction could be seen in the patterns of use of visualization and associated emotion and in the meta-emotion and beliefs of the individuals. It has been shown that the cognitive processes of visual reasoning and negative emotion interaction occur in identifying strategies of interactive representation and processing of certain representations, where the student has to put into play the identification of parametric variations. 
A distinction has been made between computer imagery and mathematical object imagery, and, through instrumental and dimensional deconstruction concepts, the existence of separate cognitive mechanisms for processing objects and relations among objects, where figurative and instrumental genesis processes are involved, has been shown.

In addition, the affective-cognitive dynamics can generally suffer sudden changes under special conditions. When the emotional tensions in mental or social systems reach a critical point, the dominant forms of feeling, thinking, and acting can suffer sudden global changes (called non-linear bifurcation). In this study, the control parameter that determines the moment of bifurcating was the meta-emotion and beliefs of subjects. Through implicative data analysis that took into account these small-scale observations as categories, it was possible to transfer to a large-scale process. As seen in Sect. 10.4, in individuals whose pathway is R3 (with alternating negative and positive emotions and optimized self-control of emotions) or individuals whose positive belief in the use of GeoGebra, both aspects may become the new relevant order-parameters (or nuclei of crystallization) around which the new global feeling-thinking-behaving patterns are organized.

The conceptualization of mathematical work can lead to an essential contribution to the methodology for the diagnosis of cognition and affect interaction. Regarding the cognitive dimension in appraisal processes, the categories of levels established have been useful for a global analysis, while the mathematical working space model has enabled a local look at how representations and images are produced. The transitions between figural, instrumental, and discursive processes have allowed us to characterize the dialectical process in the cognition and affect interaction between types of mathematical thinking. As we noted at the beginning, mathematical knowledge has specific characteristics that distinguish it from other areas of knowledge. Today, characterizing this is among the key issues around which we need to advance our knowledge.

Acknowledgements The paper's elaboration has been supported by a special action grant, EDU2013-44047-P, from the Government of Spain and a research grant Visiting Scholar Fellowship, University of California, Berkeley.

\section{References}

Bastide, R. (Ed.). (1962). Senses et usages du terme "structure". Hague: Mouton.

Brun, G., Doğuoğlu, U., \& Kuenzle, D. (2008). Epistemology and emotions. Hampshire, UK: Ashgate Publishing.

DeBellis, V. A., \& Goldin, G. A. (2006). Affect and meta-affect in mathematical problem solving: A representational perspective. Educational Studies in Mathematics, 63(2), 131-147.

Duval, R. (2005). Les conditions cognitives de l'apprentissage de la géométrie: développement de la visualisation, différenciation des raisonnements et coordination de leur fonctionnements. Annales de Didactique et de Sciences Cognitives, 10, 5-53.

Goldin, G. A. (2000). Affective pathways and representation in mathematical problem solving. Mathematical Thinking and Learning, 2(3), 209-219. 
Goldin, G. A. (2004). Problem solving heuristics, affect and discrete mathematics. ZDM-Mathematics Education, 36(2), 56-60.

Goldin, G. A. (2014). Perspectives on emotion in mathematical engagement, learning, and problem solving. In R. Pekrun \& L. Linnenbrink-Garcia (Eds.), Handbook of emotions and education (pp. 391-414). New York: Routledge.

Gómez-Chacón, I. Ma. (2000a). Matemática emocional. Los afectos en el aprendizaje matemático. (Emotional mathematics. Affects in mathematics learning). Madrid: Narcea.

Gómez-Chacón, I. Ma (2000b). Affective influences in the knowledge of mathematics. Educational Studies in Mathematics, 43, 149-168.

Gómez-Chacón, I. Ma . (2012). Affective pathways and interactive visualization in the context of technological and professional mathematical knowledge. Nordic Studies in Mathematics Education, 17(3-4), 57-74.

Gómez-Chacón, I. M. (2015). Meta-emotion and mathematical modeling processes in computerized environments. In B. Pepin \& B. Rösken-Winter (Eds.), From beliefs and affect to dynamic systems in mathematics education. Exploring a mosaic of relationships and interactions (pp. 201-226). Switzerland: Springer.

Gómez-Chacón, I. Mª (2017). Emotions and heuristics: The state of perplexity in mathematics. ZDM-Mathematics Education, 49, 323-338. https://doi.org/10.1007/s11858-017-0854-8.

Gómez-Chacón, I. Ma ., \& Figueral, L. (2007). Identité et facteur affectifs dans l'apprentissage des mathématiques. In Annales de Didactique et de Sciences Cognitives (Vol. 12. pp. 117-146). Strasbourg: IREM.

Gómez-Chacón, I. M., \& Kuzniak, A. (2013). Geometric work spaces: Figural, instrumental and discursive geneses of reasoning in a technological environment. International Journal of Science and Mathematics Education, 13(1), 201-226.

Gómez-Chacón, I. Mª, Romero, I. Ma ., \& Garcia, Mª . M. (2016). Zig-zagging in geometrical reasoning in technological collaborative environments: A mathematical working space-framed study concerning cognition and affect. ZDM-Mathematics Education, 48(6), 909-924.

Gras, R., Peter, P., Briand, H., \& Philippé, J. (1997). Implicative statistical analysis. In H. N. Ohsumi, N. Yajima, Y. Tanaka, H. Bock, \& Y. Baba (Eds.), Proceedings of the 5th Conference of the International Federation of Classification Societies (Vol. 2, pp. 412-419). New York: Springer.

Harre, R. (2009). Emotions as cognitive-affective-somatic hybrids. Emotion Review, 1(4), 294 301.

Immordino-Yang, M. H., \& Damasio, A. (2007). We feel, therefore we learn: The relevance of affective and social neuroscience to education. Mind, Brain and Education, 1(1), 3-10.

Lakatos, I. (1976). Proofs and refutations: The logic of mathematical discovery. New York: Cambridge University Press.

Lazarus, R. (1991). Emotion and adaptation. New York: Oxford University Press.

Malmivuori, M. L. (2006). Affect and self-regulation. Educational Studies in Mathematics, 63, 149-164.

Mandler, G. (1984). Mind and body. New York: Norton.

Pepin, B., \& Rösken-Winter, B. (Eds.). (2015). From beliefs and affect to dynamic systems in mathematics education. Exploring a mosaic of relationships and interactions. Switzerland: Springer.

Pekrun, R., \& Linnenbrink-Garcia, L. (2012). Academic emotions and student engagement. In S. L. Christenson, A. L. Reschly, \& C. Wylie (Eds.), Handbook of research on student engagement (pp. 259-282). New York, NY: Springer.

Piaget, J. (1981). Intelligence and affectivity: Their relationship during child development. Palo Alto: Annual Reviews. 
Schlöglmann, W. (2005). Affect and cognition-Two poles of a learning process. In C. Bergsten \& B. Grevholm (Eds.), Conceptions of mathematics. Proceedings of Norma 01 (pp. 215-222). Linköping: Svensk Förening för Matematikdidaktisk Forskning.

Schoenfeld, A. H. (1994). Mathematical thinking and problem solving. Lawrence Erlbaum. Hillsdale, NJ: Associates Inc.

Singer, F. M., \& Voica, C. (2010). In search of structures: How does the mind explore infinity? Mind, Brain and Education, 4(2), 81-93.

Zajonc, R. B. (1980). Feeling and thinking: Preferences need no inferences. American Psychologist, 35, 151-175.

Open Access This chapter is licensed under the terms of the Creative Commons Attribution 4.0 International License (http://creativecommons.org/licenses/by/4.0/), which permits use, sharing, adaptation, distribution and reproduction in any medium or format, as long as you give appropriate credit to the original author(s) and the source, provide a link to the Creative Commons license and indicate if changes were made.

The images or other third party material in this chapter are included in the chapter's Creative Commons license, unless indicated otherwise in a credit line to the material. If material is not included in the chapter's Creative Commons license and your intended use is not permitted by statutory regulation or exceeds the permitted use, you will need to obtain permission directly from the copyright holder. 